\title{
Underdetermined Blind Source Separation of FSK Signal Based on Particle Swarm Optimization
}

\author{
XIA Jiang-hua ${ }^{1,}$, , Yang $\mathrm{li}^{2, \mathrm{~b}}$ \\ ${ }^{1}$ Sichuan Aerospace Vocational College, Chengdu 610100, Sichuan, China \\ 2 Sichuan Aerospace Vocational College, Chengdu 610100, Sichuan, China \\ a170862@qq.com, b304931112@qq.com
}

\begin{abstract}
Keywords: FSK signal; Independent component analysis; Particle swarm optimization; blind signal separation.

Abstract. It's likely undetermined case that the observed signal is less than the number of source number, while the FSK signal of track circuit is mixed with a variety of interference signal. The traditional independent component analysis (ICA) cannot separate source signals. The article separate source signals use the algorithm of FSK signal underdetermined blind separation based on particle swarm optimization. Finally through the computer simulation, it show this method can effectively separate the FSK signal interference signals, and have good separation performance.
\end{abstract}

\section{Introduction}

Typically, the detection of track circuit frequency-shift signal, time domain signal filtering, spectral analysis, or can transform traditional methods such as ${ }^{[1-2]}$ But because the impact of interference signals, FSK Signal detection accuracy and reliability has been greatly restricted. Blind source separation (Blind Source Separation, BSS) method has been widely used in the fields of signal processing. The so-called blind source separation, that is, in the absence of prior knowledge of the case, solely on the basis of the statistical characteristics of the input signal, recovered from the observed mixed signal source signals. Restoration of signals and signal compared to the sort of fuzziness and amplitude scaling issues, but retain the source waveform information. Blind source separation of track circuit frequency-shift signal, some scholars made a lot of important work. Chai Xiaodong ${ }^{[3]}$, such as independent component analysis (ICA) separated by the method of interference signals; Liu ${ }^{[4]}$ presents the orthogonality constraint variable step size Adaptive blind source separation algorithm has achieved good results. But the study is in the case of definite and positive definite, namely signal vector dimension greater than or equal to the source signal vector dimension. In practical work, poorly defined the number of source signals, sensors also determines the number of distribution, but limited by cost, often have sensors smaller than the number of source signals ${ }^{[5-8]}$.

This paper introduces the sparse underdetermined blind source separation algorithm and Particle Swarm Optimization algorithm and study of clustering algorithm based on Particle Swarm Optimization algorithm to estimate the mixing matrix; increased use of particle swarm optimization algorithm for solving optimization problems with linear constraints, get the source signal and the evaluation analyzes the algorithm performance.

\section{Particle Swarm Optimization algorithm for solving optimization problems with linear constraints}

Particle Swarm Optimization algorithm for sparse component analysis

Blind signal separation based on Particle Swarm Optimization algorithm for sparse algorithms are as follows:

(1) The mixed signals $x(t) \quad(t=1,2, \mathrm{~L}, T)$ sparse transform(For example, FFT, DCT and Wavelet packet transforms) convert sparse signal, as $P(x(t)),(t=1,2, \mathrm{~L}, T)$ the transformed signals; and unified the mixed signals; 
(2) Using the clustering method based on PSO algorithm, clustering $P^{\prime}(x(t))(t=1,2, \mathrm{~L}, T)$;

(3) Clustering each class determine the direction vector $a_{1}, a_{2}, \mathrm{~L}, a_{n}$, to determine the mixing matrix $A=\left(a_{1}, a_{2}, \mathrm{~L}, a_{n}\right)$;

(4) Using PSO algorithm for solving the following problems: $\min _{P\left(s_{i}(t)\right)} \sum_{i}\left|P\left(s_{i}(t)\right)\right| \quad$ s.t. : AP(s $\left.(t)\right)=P(x(t)), t=1,2, \mathrm{~L}, T$

(5) Seperate sparse signals $P(x(t))(t=1,2, \mathrm{~L}, T)$ from a sparse field. In order to achieve source signal reconstruction with sparse transform,.

\section{Algorithm simulation}

\section{Simulation of signal representation}

FSK is the signal that discrete signals using digital signal carrier frequency shift keying modulation signal. the FSK signal that discussed in this paper is continuous phase, binary modulation commonly used in the track circuit.

Continuous phase FSK time domain expression:

$$
s 1(t)=A_{0} \cos \left[2 \pi f_{0} t+g(t)\right]
$$

In equation:

$$
g(t)= \begin{cases}2 \pi \Delta f t & 0<t<\frac{T}{2} \\ 2 \pi \Delta f\left(\frac{T}{2}-t\right) & \frac{T}{2}<t<T\end{cases}
$$

The main parameters of FSK signal is amplitude $A_{0}$, carrier frequency $f_{0}=\frac{f_{H}+f_{L}}{2}$, low modulation frequency $f_{d}=1 / T$, top frequency $f_{H}=f_{0}+\Delta f$ and bottom frequency。

$$
s 2(t)=\left[1+m \cos \left(\omega_{1} t+\varphi\right)\right] \cos \left(\omega_{2} t+\theta\right)
$$

In equation: $\omega_{1}, \omega_{2}$ is modulation frequency and carrier frequency; $m$ coefficients of modulation; $\varphi, \theta$ modulation frequency and carrier initial phase. In the field environment, it often subject to the effect of $50 \mathrm{~Hz}$ power-line interference $s 3$.

The mixed-signal mixed with interference is represented as:

$$
x(t)=s 1(t)+s 2(t)+s 3(t)
$$

At the same time 2 Set of mixed signals get from track circuits:

$$
\begin{array}{r}
x_{1}(t)=a_{1,1} s 1(t)+a_{1,2} s 2(t)+a_{1,3} s 3(t) \\
x_{2}(t)=a_{2,1} s 1(t)+a_{2,2} s 2(t)+a_{2,3} s 3(t)
\end{array}
$$

In equation: $a_{1}, a_{2}, a_{3}, a_{4}$ the strength of FSK signal and interference.

Matrix of mixed-signal show as

$$
\left[\begin{array}{l}
x_{1}(t) \\
x_{2}(t)
\end{array}\right]=\left[\begin{array}{lll}
a_{1,1} & a_{1,2} & a_{1,3} \\
a_{2,1} & a_{2,2} & a_{2,3}
\end{array}\right]\left[\begin{array}{l}
s 1(t) \\
s 2(t) \\
s 3(t)
\end{array}\right]
$$

\section{Simulation experiment}

Simulation experiment to the FSK signal, FM jamming signal, power frequency interference signals as three source, as shown in figure 2,

Mix matrix: 


$$
A=\left[\begin{array}{lll}
0.9578 & 0.5812 & 0.9285 \\
0.2873 & 0.8137 & 0.3714
\end{array}\right]
$$

By equation(1) get mixed signals $x_{1}$ and $x_{2}$, as shown in figure 3. You can see two mixed signal is not sparse signals, sparse signal $P(x(t)) \quad(t=1,2, \mathrm{~L}, T)$ is obtained by wavelet packet transform, and the scatter diagram as shown in figure 3. After normalization $P^{\prime}(x(t))$, the scatterplot by figure 4 . As can be seen that the corresponding point clustering integration is not strong, lead to confusion matrix $A$ deviation is bigger. For threshold processing $P^{\prime}(x(t))$, sparse of the signal increase, the scatter as shown in figure 5.

By clustering method based on particle optimization, estimation of the mixing matrix $A$.

$$
\hat{A}=\left[\begin{array}{lll}
0.9285 & 0.9578 & 0.3875 \\
0.3714 & 0.2873 & 0.8758
\end{array}\right]
$$

The source of the signal show in figure 6. The source signals and separation between the correlation coefficient are shown in table 1. From figure 6 shows the algorithm is effective and can be seen from table 1 of the BSS algorithm has good separation performance.

Table 1 source signals and separation between the correlation coefficient

\begin{tabular}{cccc}
\hline $\begin{array}{c}\text { Source } \\
\text { Signal }\end{array}$ & $\begin{array}{c}\text { Separate } \\
\text { Signal S1 }\end{array}$ & $\begin{array}{c}\text { Separate } \\
\text { Signal S2 }\end{array}$ & Separate Signal S3 \\
\hline S1 & 0.9997 & 0.0004 & 0.0019 \\
S2 & 0.0005 & 1.0000 & 0.0025 \\
S3 & 0.0009 & 0.0021 & 0.9996 \\
\hline
\end{tabular}

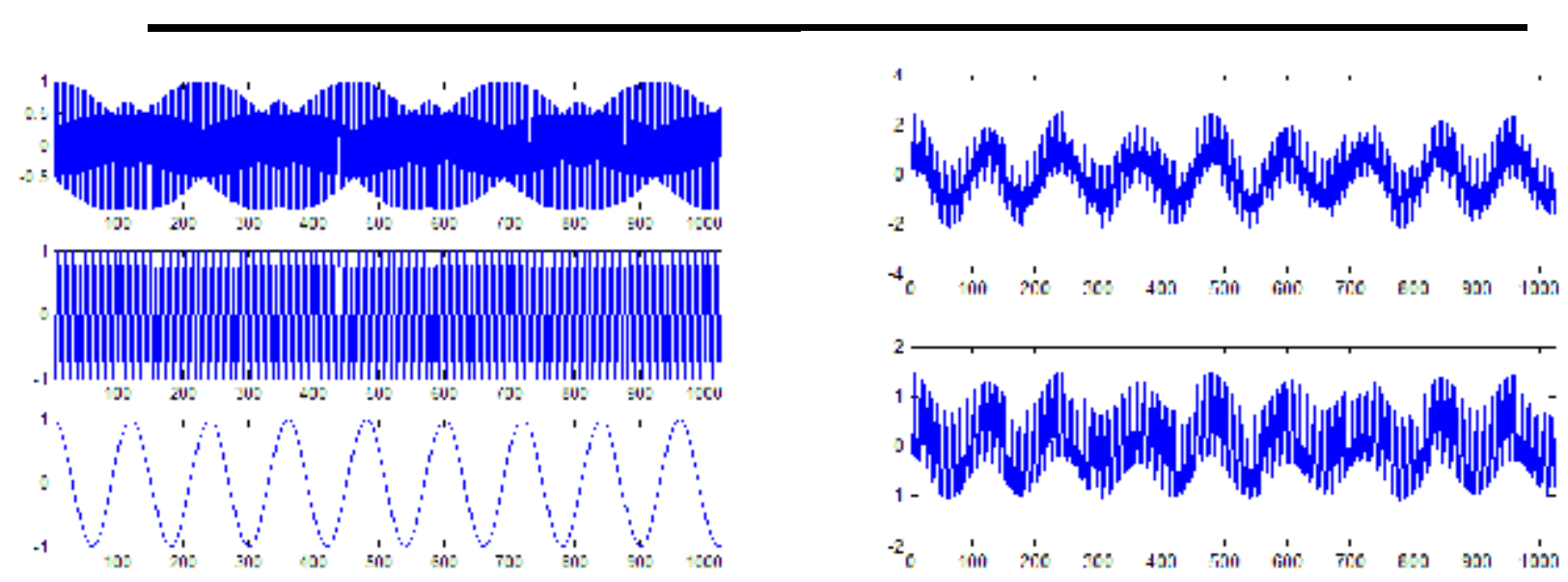

Fig1 three source signal

Fig 2 two mixing signals 


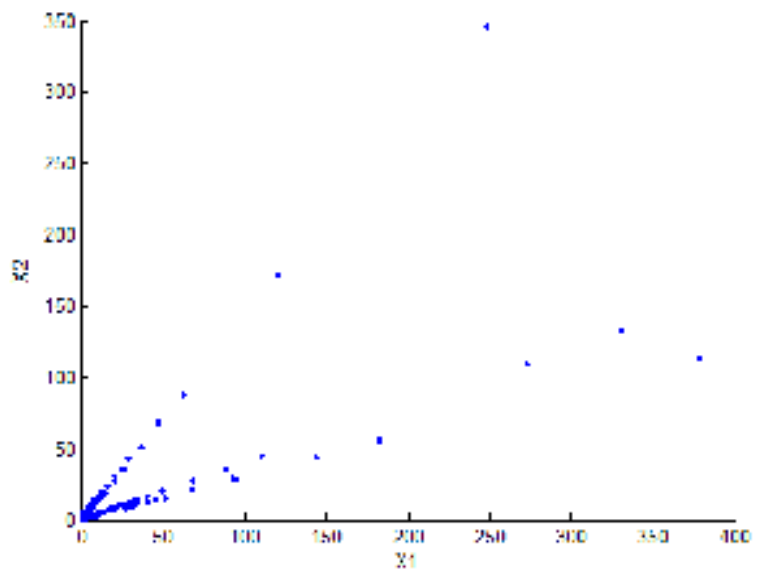

Fig 3. aliasing signal scatter plot

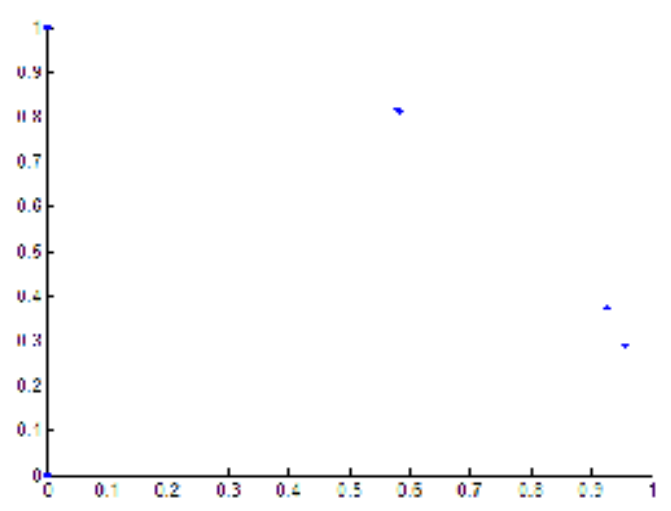

Fig 5. threshold processing mixed signal after a scatter diagram

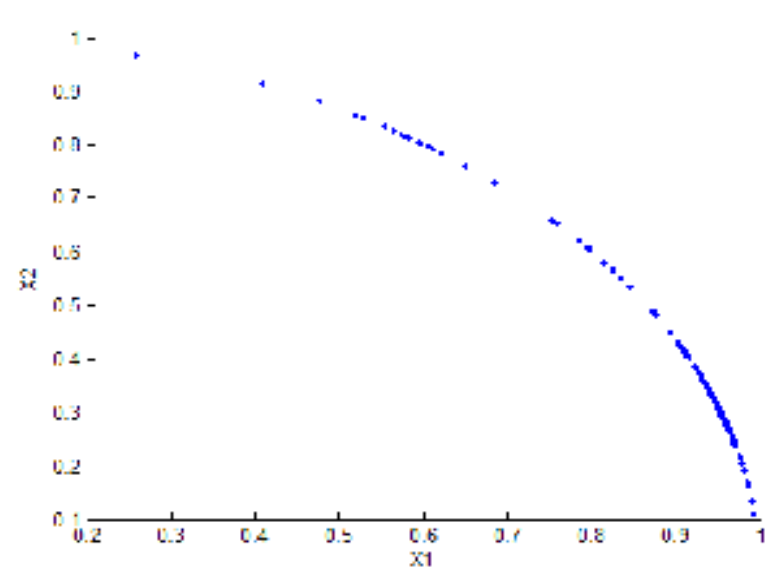

Fig 4. normalized aliasing signal scatter plot
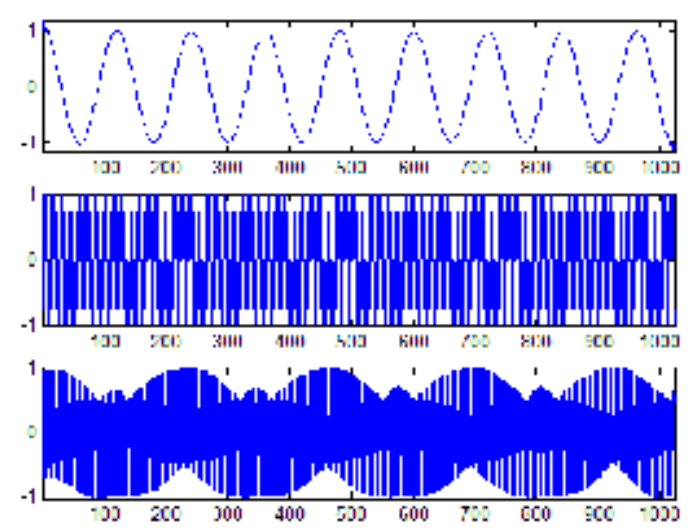

Fig6 .recovery three source signal

\section{Conclusions}

This article in view of the FSK signal detection of frequency shift track circuit may appear underdetermined, "two steps" sparse component analysis method is used for mixed signal blind source separation, and in the first step of the particle optimization algorithm is adopted to clustering algorithm is optimized, in the second step when using particle optimization algorithm with constraints was used to solve linear programming, the source signals. For mixed signal sparse transformation, set the appropriate threshold, can improve the signal sparse, and then improve the estimation precision of mixed matrix; Computer simulation results show that the method can restore the source signal, and better separation performance.

\section{References}

[1] GAO Hongmin, JIA Xexiang, ZHAO Haidong. Research of Chinese Railway Special 2FSK Modulated Signal Generating Method [J]. China Railway Science, 2002, 23 (4): 101-105. in Chinese.

[2] DENG Yinghong, XIAO Caixia. Signal Selection and Demodulation Method of Multi-Information Shifting Frequency Automatic Blocking System [J]. China Railway Science, 2002, 23 （3） : 55-59. in Chinese.

[3] CAI Xiaodong, RAO Huiming. Removal of Interference Signal from FSK Signal Using the Method of Blind Signal Separation Based on Independent Component Analysis[J]. China Railway Science, 2009, 30 (4). 
[4] LIU Ning, ZHANG Weitao. Natural Gradient Based Blind Signal Separation Algorithm with Variable Step Size and Orthogonal Constraint[J]. China Railway Science, 2010, 31 （6）.

[5] M.Zibulevsky and B. A. Pearlmutter, Blind Source Separation by Sparse Decompositon[J], Neural Comput. 13(4),pp.863-882, 2001.

[6] T. W. Lee, M. S. Lewicki, M. Girolami, and T.J. Sejnowski, Blind source separation of more sources than mixtures using overcomplete representations [J], IEEE Signal Process Letters, 6(4), pp.87-90, 1999.

[7] P.Bofill and M.Zibulevsky, Underdetermined blind source separation using sparse representations[J], Signal Process, 81(11), pp.2353-2362, 2001.

[8]Yang Hang completed, the writing; The artificial neural network and fanaticism, processing [M]. Beijing: tsinghua university press, 2003. 